\title{
Dependence of new-snow density on slope angle
}

\author{
Yasoichi Endo, Yuji Kominami, Shouji Niwano \\ Tohkamachi Experiment Station, Forestry and Forest Products Research Institute, Tohkamachi City, Niigata 948, Japan
}

\begin{abstract}
The mass per unit horizontal area, vertical height and density of new snow accumulated on various slopes of $0^{\circ}$ to $75^{\circ}$ were measured. Although the mass of new snow on these slopes was nearly the same, vertical height increased and density decreased with increase of slope angle. Differences in heights and densities of new snow due to slope angle were explained by considering both accumulation and densification processes on the slopes.
\end{abstract}

\section{INTRODUCTION}

Under windless conditions, mass per unit horizontal area, vertical height and density of new snow accumulated on slopes are usually considered to be equal to those on a horizontal surface. It was found first by Takahashi and Nogami (1952) and confirmed by Shidei (1952) that the vertical height of new snow on a slope increases with slope angle and density decreases with slope angle, though the mass per unit horizontal area is nearly the same whether on a slope or on a horizontal surface. However, this result did not receive much attention and was forgotten. The dependence of height and density on slope angle is not only essential as basic knowledge but may also play an important part in avalanche formation. Hence, we re-investigated this de-

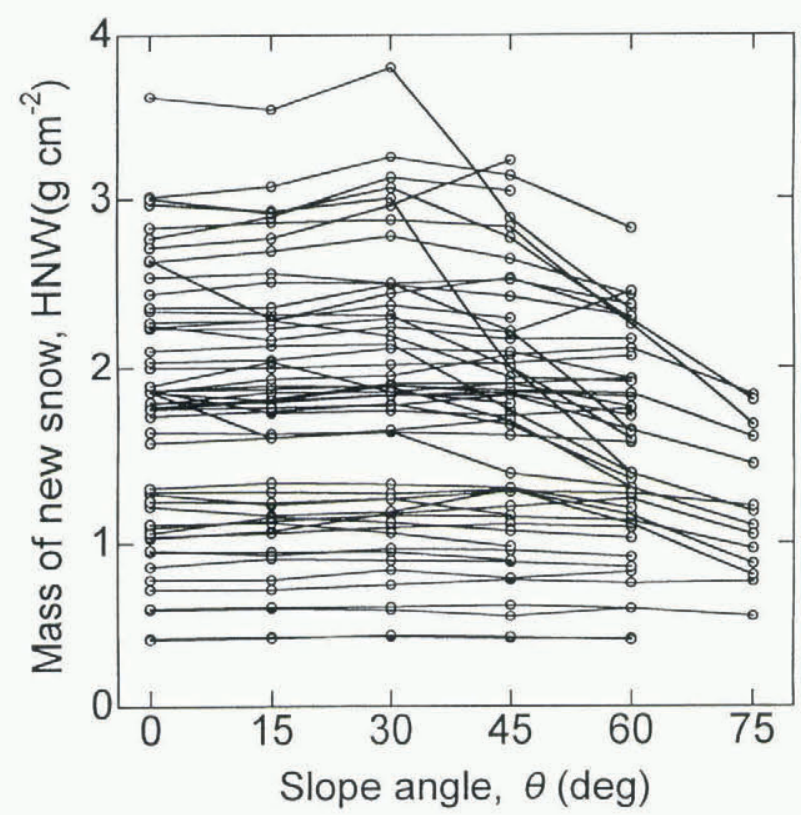

Fig. 1. Relation between mass $H N W$ of new snow per unit horizontal area and slope angle $\theta$. pendence in a field experiment. Here, the results are shown and the causes are explained.

\section{METHOD OF FIELD EXPERIMENT}

In order to measure mass, vertical height and density of new snow on various slopes, we constructed six wooden slopes $90 \mathrm{~cm}$ long and $60 \mathrm{~cm}$ wide inclined at $0^{\circ}, 15^{\circ}, 30^{\circ}, 45^{\circ}, 60^{\circ}$ and $75^{\circ}$ on flat ground at the Tohkamachi Experiment Station. They were surrounded by a sheet $3 \mathrm{~m}$ high to reduce irregular accumulation of new snow due to strong winds or sunshine. Measurements of mass $H N W$ per unit horizontal area and vertical height $H N$ of new snow accumulating on the slopes during intervals of 3 to 24 hours were made using snow samplers and measuring rulers, cut so they were parallel to the slope when inserted vertically into the snow. Mean densities $\rho$ of new snow were calculated by $\rho=H N W / H N$. This experiment was carried out during the winters of 1990-91, 1991-92 and 1992-93 in Tohkamachi.

\section{RESULTS OF FIELD EXPERIMENT}

All masses $H N W$ measured during the three winters were plotted against slope angle $\theta$ in Figure 1, where open circles connected by a solid line represent $H N W$ of new snow accumulated on each slope during a measuring interval. Variations of $H N W$ are small on slopes below $45^{\circ}$ but decrease on slopes above $60^{\circ}$, because of sliding and rolling of snow particles.

If snow falls vertically under windless conditions and accumulates on a slope without slipping down, $H N W$ should be independent of slope angle. In order to study the dependence of height and density on slope angle, we needed to investigate new snow which had accumulated uniformly on a slope without the influence of wind, sunshine or sliding. We therefore selected cases where the ratio of $H N W$ on a slope to that on a horizontal surface was within $100 \pm 5 \%$, and plotted densities $\rho$ of new snow against slope angle $\theta$. Figure 2 shows that the density $\rho$ of new snow on a slope decreases 


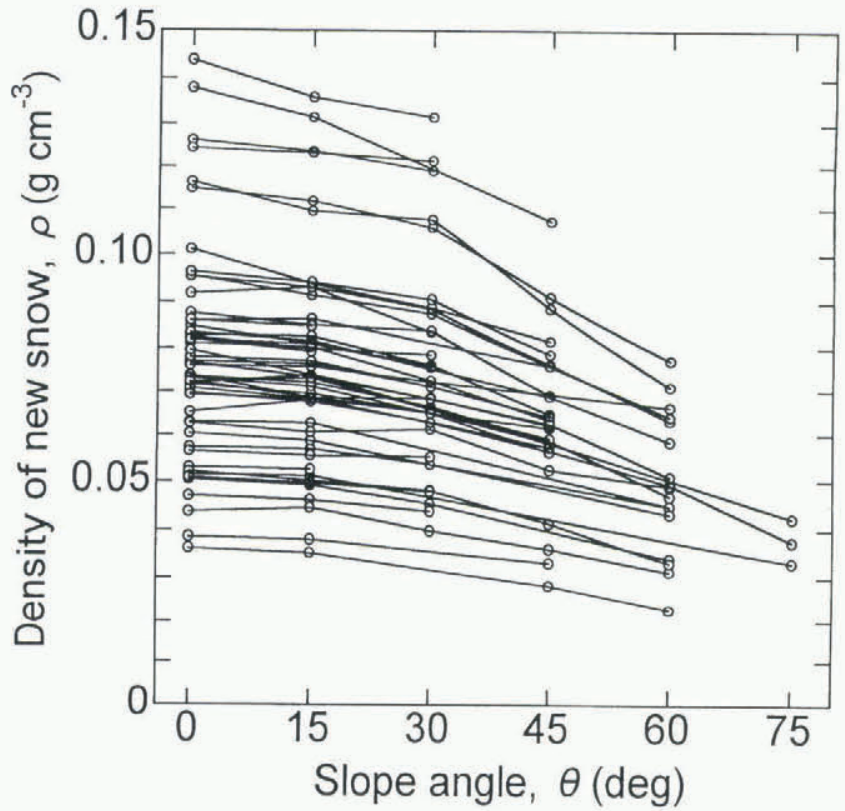

Fig. 2. Relation between density $\rho$ of new snow and slope angle $\theta$.

as the slope increases. Consequently, the vertical height $H N$, being inversely proportional to density $\rho$ for similar masses $H N W$, increases as the slope increases.

\section{DEPENDENCE OF HEIGHT AND DENSITY ON SLOPE}

In Figures 3 and 4, vertical heights $H N_{\theta}$ and densities $\rho_{\theta}$ of new snow on a slope of angle $\theta$ are plotted respectively against heights $H N_{0}$ and densities $\rho_{0}$ on a horizontal surface $\theta=0$. These figures show that $H N_{\theta}$ and $\rho_{\theta}$ respectively are well approximated by the following straight lines

$$
H N_{\theta}=(1 / B) H N_{0} \text { and } \rho_{\theta}=B \rho_{0} .
$$

Here, the mean values of $B$ obtained from the straight lines

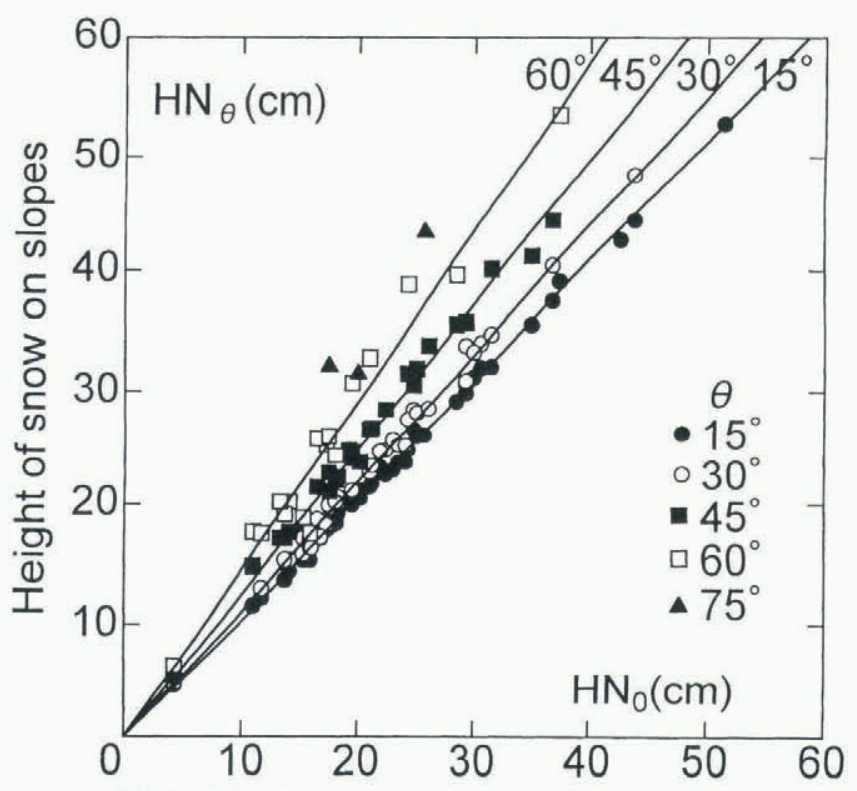

Height of snow on a horizontal surface

Fig. 3. Relation between vertical height $H N_{\theta}$ of new snow on on a slope and height $H N_{0}$ on a horizontal surface.

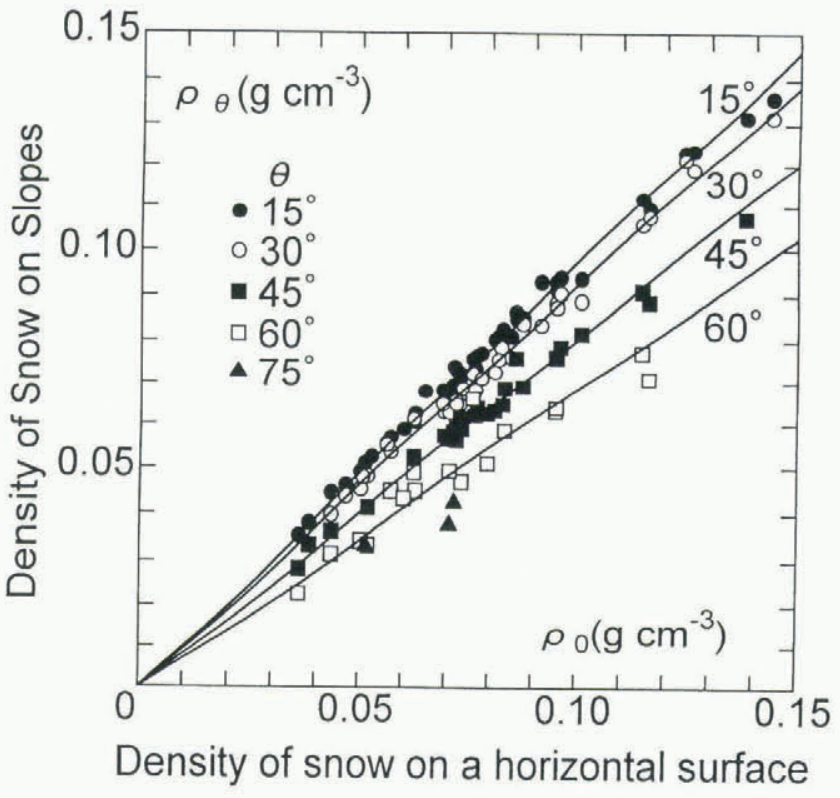

Fig. 4. Relation between density $\rho_{\theta}$ of new snow on a slope and density $\rho_{0}$ on a horizontal surface.

in Figures 3 and 4 are 0.975 for $\theta=15^{\circ}, 0.917$ for $\theta=30^{\circ}$, 0.804 for $\theta=45^{\circ}$ and 0.687 for $\theta=60^{\circ}$. Then, plotting the obtained values of $\log B$ against $\log (\cos \theta)$, we obtained the following relation

$$
B=\rho_{\theta} / \rho_{0}=H N_{0} / H N_{\theta}=(\cos \theta)^{\alpha}
$$

with $\alpha=0.57$ for new snow accumulated during measuring intervals of 3-24 hours. The solid curve in Figure 5 is the plot of Equation (1), while open circles are the plots of $B$

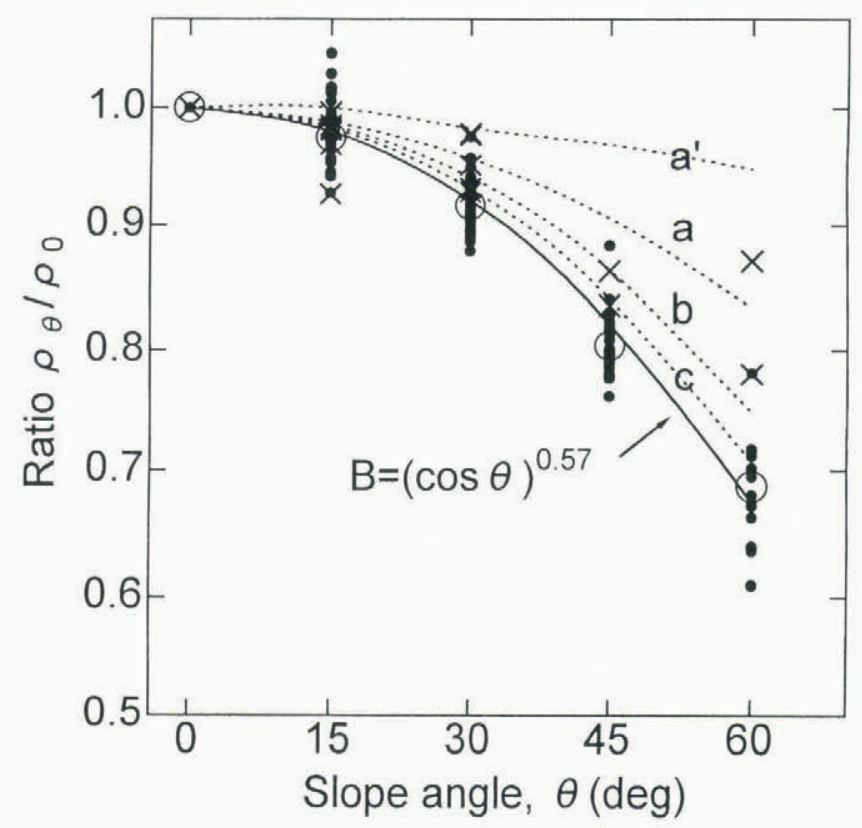

Fig. 5. Relation between the ratio $\rho_{\theta} / \rho_{0}$ and slope angle $\theta$. Open circles are the values $B$ obtained from straight lines in Figures 3 and 4 ; crosses mark the ratios $\rho_{\theta} / \rho_{0}$ for the 3-7hour intervals; solid circles those for the 17-24 hour intervals. The solid curve is the plot of Equation (1); the broken curve $a^{\prime}$ the plot of $\rho_{\theta} / \rho_{0}$ computed for $\alpha_{0}=0$ over an interval of 7 hours; and broken curves $a, b$ and $c$ the plots of $\rho_{\theta} / \rho_{0}$ for $\alpha_{0}=0,0.3$ and 0.5 over a 24 hour interval. 
against $\theta$. As shown in Figure 5, the values of $B$ are well approximated by Equation (1).

To investigate the influence of the length of the measuring interval, we plotted the ratios $\rho_{\theta} / \rho_{0}$ obtained for each measuring interval against $\theta$ in Figure 5 , where crosses are for the intervals of 3-7 hours; and solid circles are for those of 17-24 hours. Although the data for the intervals of 3-7 hours are few, most are distributed above the plot of Equation (1). That is, the value $\alpha$ for the short intervals of 3-7 hours, ranging from $\alpha=0.2$ to 0.5 , is smaller than the value for those of 17-24 hours. Such an increase of $\alpha$ with the length of measuring interval suggests that the dependence of density on slope is caused partly by differences in snow densification on slopes.

\section{DENSIFICATION OF SNOW ON SLOPES}

Studies based on viscous compression models to estimate the density profile of a snow cover have been conducted successfully by Kojima (1967), Endo (1992) and others. We computed densities of new snow on various slopes to compare with measured values, using the following empirical relation between compressive viscosity $\eta$ and density $\rho$ found for snow with a density range of between 40-300 $\mathrm{kg} \mathrm{m}^{-3}$ by Endo and others (1990):

$$
\eta=C \rho^{\mathrm{n}}
$$

where $\mathrm{C} \doteqdot 0.392 \mathrm{~Pa} \mathrm{~s}\left(\mathrm{~kg} \mathrm{~m}^{-3}\right)^{-\mathrm{n}}$ and $\mathrm{n} \doteqdot 4.0$.

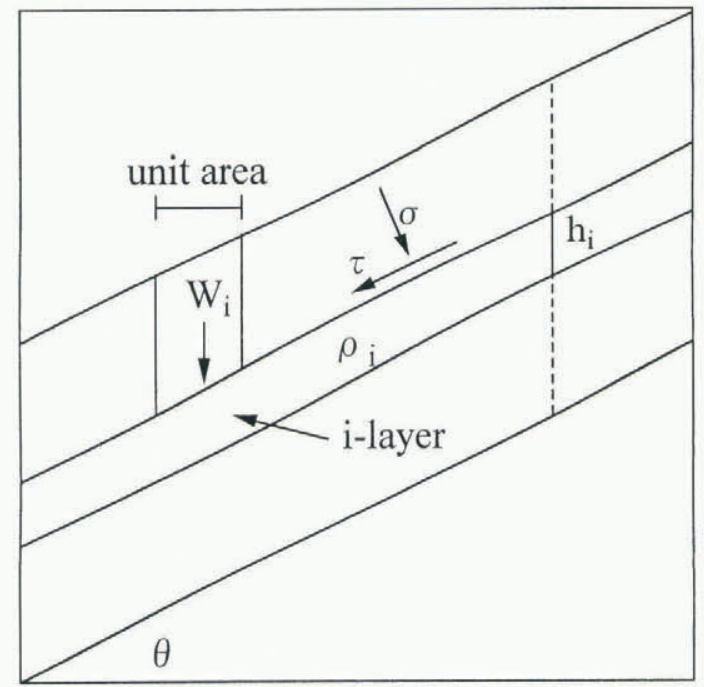

Fig. 6. Model of a snow cover on a slope.

Now we consider a thin snow layer called $i$ layer which is accumulated at time $t_{i}$, as shown in Figure 6. Let $h_{i}(t)$ and $\rho_{i}(t)$ be vertical thickness and density of $i$ layer at time $t$, and $W_{i}(t)$ be weight per unit horizontal area of snow lying on $i$ layer at time $t$. Then, normal stress and shear stress exerted on $i$ layer are respectively $\sigma=W_{i}(t)(\cos \theta)^{2}$ and $\tau=W_{i}(t) \cos \theta \sin \theta$. The $i$ layer is compressed in the normal direction by $\sigma$, and sheared parallel to the slope by $\tau$ which does not affect a thickness of $i$ layer. If $i$ layer is compressed by $\sigma$ to have a thickness $h_{i}-\mathrm{d} h_{i}$ and a density $\rho_{i}+\mathrm{d} \rho_{i}$ in a short time interval $\mathrm{d} t$, a compressive strain rate $\dot{\varepsilon}$ in the direction normal to $i$ layer is given by $\dot{\varepsilon}=-\left(1 / h_{i}\right)(\mathrm{d} h i / \mathrm{d} t)=\left(1 / \rho_{i}\right)\left(\mathrm{d} \rho_{i} / \mathrm{d} t\right)$. As snow is a vishttps://doi.org/10.3189/1998AoG26-1-14-18 Published online by Cambridge University Press cous compressive material, we assume that the relation between $\sigma$ and $\dot{\varepsilon}$ is given by $\sigma=\eta \dot{\varepsilon}$. Then, we have

$$
W_{i}(t)(\cos \theta)^{2}=\eta\left(1 / \rho_{i}\right)\left(\mathrm{d} \rho_{i} / \mathrm{d} t\right) .
$$

Substituting Equation (2) into Equation (3) and integrating both sides after separating the variables, we have as an equation giving the time variation of snow density

$$
\rho_{i, \theta}(t)=\left[(\mathrm{n} / C)(\cos \theta)^{2} Q_{i}\left(t_{i}, t\right)+\left(\rho_{\text {init }, \theta}\right)^{\mathrm{n}}\right]^{\frac{1}{n}}
$$

where $\rho_{i, \theta}(t)$ is the snow density of $i$ layer on a slope $\theta$ at time $t, \rho_{\text {init, } \theta}$ the initial density on a slope $\theta$, and $Q_{i}\left(t_{i}, t\right)$ the time-integration of weight per unit horizontal area of snow lying on $i$ layer from $t_{i}$ to $t$ :

$$
\begin{aligned}
Q_{i}\left(t_{i}, t\right)=\int_{t_{i}}^{t} W_{i}(t) \mathrm{d} t & =\int_{t_{i}}^{t}\left(t-t_{s}\right) \mathrm{d} W_{s} \\
& =\int_{t_{i}}^{t}\left(t-t_{s}\right) q\left(t_{s}\right) g \mathrm{~d} t_{s}
\end{aligned}
$$

where $W_{i}(t)$ is a weight per unit horizontal area of snow lying on $i$ layer at time $t, \mathrm{~d} W_{s}=q\left(t_{s}\right) g \mathrm{~d} t_{s}$ a weight of $s$ layer accumulated from time $t_{s}$ to $t_{s}+\mathrm{d} t_{s}, q\left(t_{s}\right)$ an accumulation rate at time $t_{s}$, and $g$ is gravitational acceleration. Consequently, if hourly precipitation from time $t_{i}$ up to $t$ is known, we can compute numerically the density $\rho_{i, \theta}(t)$ of $i$ layer on a slope $\theta$ at time $t$ from Equations (4) and (5).

Since the vertical thickness $h_{i, \theta}(t)$ of $i$ layer on a slope $\theta$ at time $t$ is given by $q\left(t_{i}\right) \mathrm{d} t_{i} / \rho_{i, \theta}(t)$, the vertical height $H N_{\theta}$ of new snow accumulated on a slope $\theta$ from time $t_{i}$ to $t$ is given by

$$
H N_{\theta}=\int_{t_{i}}^{t}\left\{q\left(t_{s}\right) / \rho_{s, \theta}(t)\right\} \mathrm{d} t_{s}
$$

and the mean density $\rho_{\theta}$ of the new snow on a slope $\theta$ becomes

$$
\rho_{\theta}=\left[\int_{t_{i}}^{t} q\left(t_{s}\right) \mathrm{d} t_{s}\right] / H N_{\theta} .
$$

Now consider a case that $Q_{i}\left(t_{i}, t\right)$ satisfies the following condition:

$$
Q_{i}\left(t_{i}, t\right) \gg(C / \mathrm{n})(\cos \theta)^{-2}\left(\rho_{\text {init }, \theta}\right)^{\mathrm{n}} .
$$

Then, Equation (4) becomes

$$
\rho_{i, \theta}(t)=\left\{(\mathrm{n} / C)(\cos \theta)^{2} Q_{i}\left(t_{i}, t\right)\right\}^{\frac{1}{n}}=(\cos \theta)^{\frac{2}{n}} \rho_{i, 0}(t)
$$

where $\rho_{i, 0}(t)$ is a density of $i$ layer on a horizontal surface $\theta=0$ at time $t$. If most new snow layers accumulated from time $t_{i}$ to $t$ satisfy Equation (8), the ratio $\rho_{\theta} / \rho_{0}$ for the mean density of the snow is given by

$$
B=\rho_{\theta} / \rho_{0}=H N_{0} / H N_{\theta}=(\cos \theta)^{\frac{2}{n}} .
$$

This result indicates that the value of $\rho_{\theta} / \rho_{0}$ approaches Equation (9) unrelated to the initial snow density, as the measuring interval is long. Since the value $\mathrm{n}$ is about 4 according to Endo and others (1990), Equation (9) is nearly equal to Equation (1) obtained for the measuring intervals of 3-24 hours. However, it is not certain whether our measurements satisfy Equation (8).

In order to study that relationship, we computed mean densities $\rho_{\theta}$ of new snow accumulated under constant rates 
on various slopes. In the computations, we assumed that the initial density $\rho_{\text {init }, \theta}$ was related to the initial density $\rho_{\text {init, } 0}$ on a horizontal surface by

$$
\rho_{\text {init }, \theta} / \rho_{\text {init }, 0}=(\cos \theta)^{\alpha_{0}}
$$

where $\alpha_{0}$ is $\alpha$ for the initial density, using the following values: $q\left(t_{s}\right)=1.0 \mathrm{~kg} \mathrm{~m}^{-2} \mathrm{~h}^{-1}\left(=1.0 \mathrm{~mm}\right.$ water $\left.^{-1}\right), \rho_{\text {init }, 0}=$ $50 \mathrm{~kg} \mathrm{~m}^{-3}, \alpha_{0}=0,0.3$ and 0.5 , time interval equals 7 and 24 hours. The computed ratios $\rho_{\theta} / \rho_{0}$ are shown by broken curves in Figure 5, where the curve a' represents the relation for $\alpha_{0}=0$ over a 7 hour interval and the curves a, b and c represent relations for $\alpha_{0}=0,0.3$, and 0.5 over an interval of 24 hours.

As shown in Figure 5, the observed data are best represented by the broken curves b and c for $\alpha_{0}=0.3$ and 0.5 , and hence we can say that the observed difference of snow density due to slopes can be explained by the process of snow densification in cases where $\alpha_{0}$ ranges from 0.3 to 0.5 , but not in cases where $\alpha_{0}=0$. When $\alpha_{0}=0$, the initial snow density just after accumulation does not vary with slope angle.

\section{DIFFERENCE OF INITIAL SNOW DENSITY DUE TO SLOPE}

In order to investigate differences of initial snow density on various slopes, we measured densities of the surface snow lying up to $3 \mathrm{~cm}$ below the surface directly with a snow sampler, $3 \mathrm{~cm}$ high, $6 \mathrm{~cm}$ wide and $5.5 \mathrm{~cm}$ long. The results are shown by open circles in Figure 7, where the numeral in parentheses by the open circle shows the time which passed from accumulation of snow to its measurement. Figure 7 indicates that densities of new snow accumulated before 1 or 5 hours depend on slope angle; and the values of $\alpha$ range from 0.2 to 0.5 . Such a dependence just after snow accumulation must be caused by the process of accumulation of snow crystals or snowflakes on the slopes.

From these results, we can say that the dependence of snow density on slope angle originates in the process of both accumulation and densification of snow on the slopes; the value of $\alpha$, ranging from 0.2 to 0.5 for the initial snow densities just after accumulation, comes to about 0.56 for the mean densities of new snow accumulated during about 1 day. Theoretically, the value $\alpha$ is considered to approach $\frac{2}{n}$ with n equal about 4 , as the lapse of time is long enough to satisfy Equation (8).

\section{PROGESS OF SNOW ACGUMULATION ON SLOPES}

Here we discuss the dependence of initial snow density on slope angle. The shape of new snow can be classified into plate-like crystals such as plates and stellar crystals, needlelike crystals such as needles and columns and sphere-like crystals such as spatial dendrites and graupel. Accumulation of plate-like and needle-like crystals on slopes is considered to be different from that of sphere-like crystals. As shown in Figure 8, plate-like crystals tend to accumulate parallel to a slope under windless condition. Then, if we assume that all plate-like crystals (including needles) accumulate parallel to slopes, the relation between vertical height $H N_{\theta}$ and $H N_{0}$ of new snow composed of plate-like crystals is given by $H N_{\theta}=(1 / \cos \theta) H N_{0}$. On the other hand, sphere-like crystals are considered to accumulate on slopes at random unrelated to slope angle, $H N_{\theta}$ for sphere-

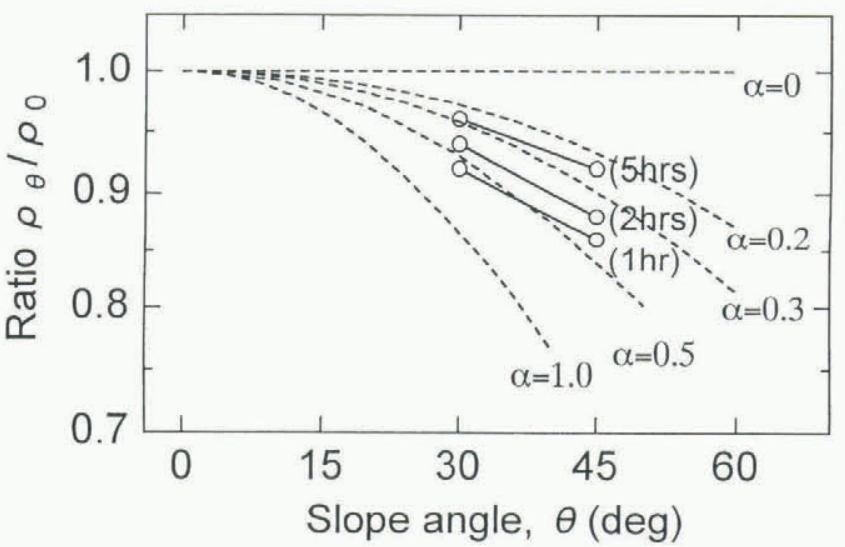

Fig. 7. Relation between the ratio $\rho_{\theta} / \rho_{0}$ and slope angle $\theta$ for the surface snow just after accumulation.

like crystals is equal to $H N_{0}$. Hence, dividing the height of new snow on a horizontal surface into two parts of plate-like and sphere-like crystals, and denoting a ratio of plate-like crystals to total height $\alpha^{\prime}$, we have the following equations as those giving the difference of height and density just after snow accumulation

$$
\begin{aligned}
H N_{\theta} & =\left\{\left(1-\alpha^{\prime}\right)+\alpha^{\prime} / \cos \theta\right\} H N_{0} \doteqdot(\cos \theta)^{-\alpha^{\prime}} H N_{0} \\
\rho_{\theta} & =\left\{\left(1-\alpha^{\prime}\right)+\alpha^{\prime} / \cos \theta\right\}^{-1} \rho_{0} \doteqdot(\cos \theta)^{\alpha^{\prime}} \rho_{0} .
\end{aligned}
$$

Takahashi and Nogami (1951) observed snow crystals of about 10 pieces falling at $1000 \mathrm{~h}$ and $1600 \mathrm{~h}$ every day during the winter of 1950-51 in Tohkamachi, and showed that $38 \%$ of the crystals were plate-like including needle-like crystals. Though the ratio of plate-like crystals is high in number, the ratio $\alpha^{\prime}$ of those to the total height of new snow may be less

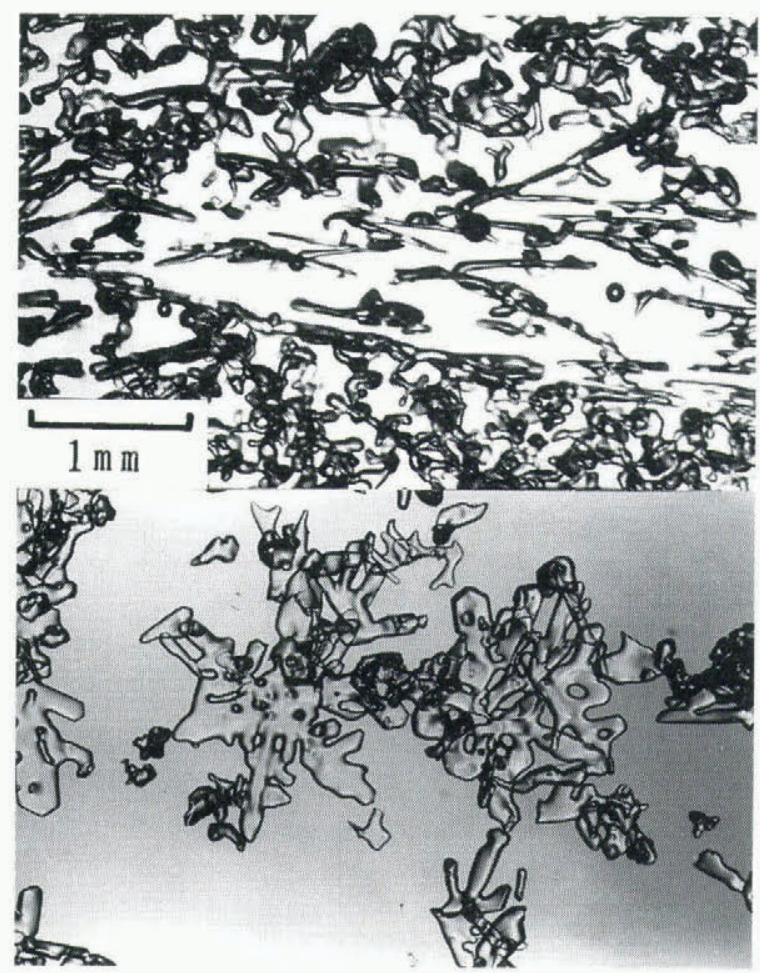

Fig. 8. Plate-like crystals accumulated parallel to a slope. Photo by Eizi Akitaya. 


\section{Snowflakes}

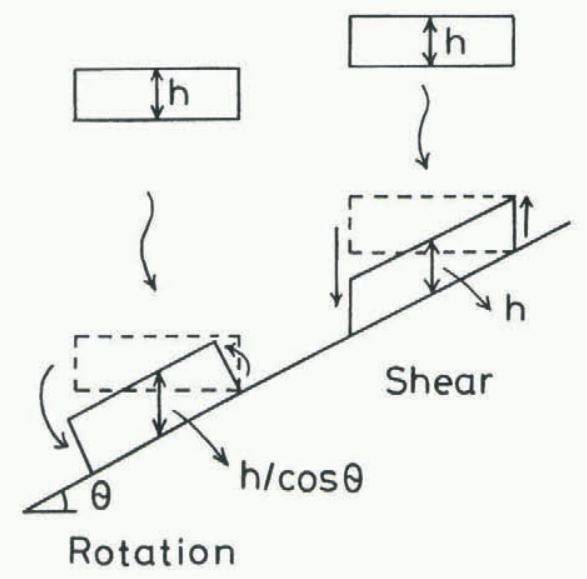

Fig. 9. Supposed accumulation process of snowflakes by rotation and shear deformation.

than 0.1 because the thickness of plate-like crystals is much smaller than the diameter of sphere-like crystals.

In Tohkamachi, snow falls as snowflakes more than as individual snow crystals. Often large snowflakes fall horizontally through the air. Though snowflakes often break when landing on a slope, they and their fragments are considered to lie parallel to the slope by their rotation and shear deformation, as shown in Figure 9. When all snowflakes lie on slopes by their rotation, the relation between $H N_{\theta}$ and $H N_{0}$ is given by $H N_{\theta}=(1 / \cos \theta) H N_{0}$. In the process of shear deformation, $H N_{\theta}$ is equal to $H N_{0}$. Then, if the probability $\alpha^{\prime}$ of rotation on a slope is given, the dependence of height and density just after the accumulation is given by Equation (11). However, it is unknown whether rotation and shear deformation take place on a slope or not.
The dependence of initial snow density on slope angle is considered to be caused by the accumulation processes of new snow as mentioned above.

\section{CONCLUSIONS}

We have confirmed that the vertical height of new snow on a slope increases with increasing slope angle and density decreases with slope angle. The weight of new snow per unit horizontal area does not vary much with slope. In addition, we have shown that the ratio of snow density on a slope to that on a horizontal surface can be expressed as a power function of the cosine of the slope angle and that such a dependence of snow density on slope angle is caused by the processes of both accumulation and densification of snow on the slope.

\section{REFERENCES}

Endo, Y. 1992. Time variation of stability index in new snow on slopes. In Proceedings. Japan - U.S. Workshop on Snow Avalanche, Landslide, Debris Flow Prediction and Control, 30 September-2 October 1991, Tsukuba, Japan. Science and Technology Agency. National Research Institute for Earth Science and Disaster Prevention, 85-94.

Endo, Y., Y. Ohzeki and S. Niwano. 1990. [Relation between compressive viscosity and density of low-density snow.] Seppyo, J. Jpn. Soc. Snow Ice, 52(4), 267-274. [In Japanese with English summary.]

Kojima, K. 1967. Densification of seasonal snow cover. In Ōura, H., ed. Physics of snow and ice. Vol. 1, Part 2. Sapporo, Hokkaido University. Institute of Low Temperature Science, 929-952.

Shidei, T. 1952. [Snow cover on slopes.] Ringyo Shikenjo Kenkyu Hokoku [Bulletin of the Forestry and Forest Products Research Institute] 54, 133-139. [In Japanese.]

Takahashi, K. and K. Nogami. 1951. [Observation of snow crystals in Tohkamachi.] Fuki, 7, 45-49. [InJapanese.]

Takahashi, K. and K. Nogami. 1952. [Metamorphism of snow on slopes.] Tuki, 10, 13-18. [In Japanese.] 